\section{A Low-Density Protein Microarray Device for Detecting Three Antibodies Associated with Invasive Fungal Infections}

\section{Abstract}

The frequency of invasive fungal infections has risen dramatically in recent years, and early and accurate diagnosis of these infections is important for appropriate use of antifungal agents. The value of serological tests is apparent, but they suffer from disadvantages, such as being time-consuming and their inability to produce simultaneous results. Protein microarrays are a convenient technology that can be used to perform high-throughput antibody detection and enable the monitoring of different antibodies simultaneously. We constructed a low-density microarray using purified recombinant enolase (Eno) fructose-bisphosphate aldolase (Fba1) from Candida and thioredoxin reductase (TR) from Aspergillus fumigatus as immobilized antigens. Following optimization of microarray construction and serum testing, we investigated the sensitivity, repeatability, and stability of the assay using sera from patients with invasive Candida and/or Aspergillus infections and control subjects. Sera from patients with invasive candidiasis (IC) were subjected to low-density microarray assay for detection of two antibodies from Candida and one antibody from $A$. fumigatus. Our results indicated a sensitivity and specificity of $67.6 \%$ and $96.5 \%$ for anti-Eno and $64.8 \%$ and $90.3 \%$ for antiFba1 antibodies, respectively. The combined detection of anti-Eno and antiFba1 returned a sensitivity of $81 \%$, and in invasive aspergillosis (IA) patients, the sensitivity and specificity of detection were $71.4 \%$ and $98.2 \%$, respectively, for the anti-TR antibody. The low-density protein microarray assay developed in this study was capable of detecting three antibodies against two fungi simultaneously, demonstrating its potential value in diagnosing IC and IA.

Keywords: Low-density protein microarray; Antibodies; Candida; Aspergillus

\section{Xiao Chen, Fang-qiu Li*, Chun-fang Ma, Li-ning Shi and Yu-an Hu}

Laboratory of Molecular Biology, Institute of Medical Laboratory Sciences, Jinling Hospital, School of Medicine, Nanjing University, Nanjing, 210002, PR China

Corresponding author: Fang-qiu Li

njlifq@163.com

Laboratory of Molecular Biology, Institute of Medical Laboratory Sciences, Jinling Hospital, 305 East Zhongshan Road, Nanjing 210002, P. R. China.

Tel: +86-13801583645

Citation: Chen X, Li F, Ma C, et al. A LowDensity Protein Microarray Device for Detecting Three Antibodies Associated with Invasive Fungal Infections. Med Mycol Open Access. 2016, 2:3.

Received: August 12, 2016; Accepted: September 29, 2016; Published: September 30, 2016

\section{Introduction}

Invasive fungal infections (IFIs) remain a major cause of mortality in those undergoing hematopoietic stem cell or solid-organ transplantation, chemotherapy for hematological malignancies, patients in intensive care units, and those infected with human immunodeficiency virus. Invasive candidiasis (IC) and invasive aspergillosis (IA) are associated with high mortality rates [1]. Candida spp. Are found in the environment in soil and water and frequently colonize human skin and mucosal membranes, with endogenous colonization responsible for most IC cases. Candidiasis covers infections that range from superficial mucosal (such as vagina) to systemic and potentially life-threatening diseases [2,3]. Aspergillus fumigatus is a saprophytic mold responsible for life-threatening, invasive pulmonary diseases in immunocompromised and in some immunocompetent hosts [4].

Early and accurate diagnosis of IFIs are important for several reasons, including timely initiation of antifungal therapy and to decrease the unnecessary use of toxic antifungal agents $[5,6]$. Unfortunately, the lack of specific symptoms and signs continues to be a major obstacle to the successful IFI treatment, and currently there is a lack of sensitive and specific methods for the early diagnosis of IFIs.

Currently, commonly used diagnostic methods, including fungal microscopy and culture, histopathologic detection, and biopsy, constitute the gold-standard methods of infection detection, but these methods are far from ideal [4]. Therefore, 
use of serological-detection methods for the early diagnosis of fungal antigens associated with IFIs continues to increase as an attractive technique to augment standard diagnostic tools [7]. Alternative serological-detection techniques include the detection of specific antigens using commercially available tests to detect galactomannan and 1, $3 \beta$-D-glucan and genomic DNA sequencing [8]. Such indirect tests (the $G$ test to detect the fungal cell-wall component (1-3)- $\beta$-D-glucan and the GM test to detect the Aspergillus cell-wall component galactomannan) are noninvasive, easy to handle, and can be conducted within hours. Although the value and significance of these methods are apparent, they suffer from several disadvantages due to their time-consuming nature, requirement of large quantities of both sample and reagents, and inability to produce simultaneous results.

Low-density microarrays allow rapid, simple, and parallel detection of many elements in a single assay that can be used to perform high-throughput detection and offer great potential for disease diagnosis [9-11]. Multiple antigens can be immobilized on a slide or a membrane as capture probes to facilitate the monitoring of a number of antibodies simultaneously. Previous studies showed that Candida albicans enolase (Eno) and fructosebisphophate aldolase (Fba1) are major immunodominant antigens in IC patients and animal models, whereas Aspergillus thioredoxin reductase (TR) is the immunodominant antigen in patients infected with Aspergillus [12-14]. Here, we developed a low-density microarray assay by immobilizing these three fungal proteins onto a nitrocellulose filter and investigated the performance of serological diagnosis of invasive infection with Candida spp. and A. fumigatus.

\section{Materials and Methods}

\section{Generation of recombinant protein}

Recombinant Eno, Fba1, and TR were prepared by genetic engineering technology using methods previously described $[14,15]$. The recombinant plasmids were transformed into Escherichia coli BL21 (DE3) cells (Novagen; Merck Millipore, Billerica, MA, USA). Expression of recombinant antigens was induced by isopropyl- $\beta-D$ - thiogalactopyranoside, and $6 x$ Histagged recombinant proteins were purified from cell-free supernatants by chromatography on $\mathrm{Ni}^{2+}$-nitrilotriacetic acidagarose. The nitrocellulose membrane, human IgG, colloidal gold-labeled goat anti-human IgG, protein-microarray spotting instrument, and PBTX-protein microarray scanner were provided by Nanjing Potomac Biotechnology Co. Ltd. (Nanjing, China).

\section{Serum collection}

All patients were admitted to Jingling Hospital, Nanjing, China. Serum samples were collected from 105 clinically diagnosed IC patients and 42 patients with culture- and/or histology documented IA from August 2010 to January 2015. According to enzyme-linked immunosorbent assay (ELISA) results, most of the patients exhibited strong antibody reactivity to Eno, Fba1, and TR. Clinically diagnosed IC was defined as the isolation of Candida spp. from the bloodstream in at least one blood culture, and proven IA patients were classified based on the standardized
IFIs Group of the European Organization for the Research and Treatment of Cancer Mycoses Study Group case definitions [16]. Serum samples from healthy volunteers and patients without clinical or microbiological evidence of IC or IA were evaluated as controls to provide data on assay specificity. Fifty serum samples were collected from people with Candida colonization (positive Candida spp. culture from sputum only), 35 serum samples were collected from bacteremia patients or patients with other fungal infections, and 183 serum samples were collected from healthy controls. All blood samples were centrifuged within $24 \mathrm{~h}$ and stored at $-70^{\circ} \mathrm{C}$ until use. The study protocol was approved by the Ethics Committee of Jingling Hospital, and informed consent was obtained from all patients included in the study.

\section{Protein-microarray assay}

The protocols used in the microarray assays for serum testing were optimized in our laboratory and included loading concentration and loading buffer for the three antigens, antigenimmobilized conditions, blocking buffer, and dilution of patient sera. According to the optimal conditions, protein-microarray devices were constructed by Nanjing Potomac Biotechnology Co. Ltd.

For serum testing, $300 \mu \mathrm{L}$ washing buffer $(0.01 \mathrm{M}$ phosphatebuffered saline with $0.1 \%(\mathrm{v} / \mathrm{v})$ Tween-20 (PBS-T) ( $\mathrm{pH} 7.4)$ ) was applied to the reaction chamber to moisten the nitrocellulose membranes, followed by the application of $80 \mu \mathrm{L}$ serum for antibody-antigen reaction. After serum passed through the nitrocellulose membrane, it was rinsed with $300 \mu \mathrm{L}$ washing buffer, followed by the addition of $400 \mu \mathrm{L}$ immunogold (colloidal gold-conjugated secondary antibody). After immunogold passed through the membrane, excess immunogold antibody was washed with $400 \mu \mathrm{L}$ washing buffer, and after $20 \mathrm{~min}$, the microarray devices were loaded into the PBT-X4 scanner and analyzed using the software Smartboy 3.0 (Nanjing Potomac Biotechnology Co. Ltd.). Red spots on the membrane indicating antibody-antigen reactions were scanned by the microarray scanner and converted to gray values using the software.

\section{Statistical analysis}

Variables without a normal distribution were expressed as median and interquartile ranges (25th-75th), and comparisons between two groups were performed using the Mann-Whitney $U$ test. The potential of the three specific antibodies to be used as diagnostic markers was tested using receiver-operating characteristic (ROC) curves with calculated area under the ROC curves (AUC). The chi-squared test was used to compare the two detection methods. All tests were two-tailed, and a $P<0.05$ was considered statistically significant. Statistical analysis was performed using SPSS 18.0 for Windows (SPSS, Chicago, IL, USA).

\section{Results}

\section{Optimization of reaction conditions}

Purified recombinant Eno, Fba1, and TR were diluted with three buffers as shown in Figure 1, showing that immobilization using Tris- $\mathrm{HCl}(14.7 \mathrm{~mL} 0.1 \mathrm{~mol} / \mathrm{L} \mathrm{HCl}$ added to $50 \mathrm{~mL} 0.1 \mathrm{~mol} / \mathrm{L}$ Tris, $\mathrm{pH} 8.5$ ) was superior to results observed using PBS ( $\mathrm{NaCl} 8 \mathrm{~g}$, 
$\mathrm{Na}_{2} \mathrm{HPO}_{4} \cdot 12 \mathrm{H}_{2} \mathrm{O} 2.08 \mathrm{~g}, \mathrm{KCl} 0.2 \mathrm{~g}, \mathrm{KH}_{2} \mathrm{PO}_{4} 0.2 \mathrm{~g}$, add distilled water to $1 \mathrm{~L}, \mathrm{pH} 7.4)$ or citrate-buffered saline $\left(\mathrm{Na}_{2} \mathrm{CO}_{3} 1.59 \mathrm{~g}, \mathrm{NaHCO}_{3}\right.$ $2.93 \mathrm{~g}$, add distilled water to $1 \mathrm{~L}, \mathrm{pH}$ 9.6). Following antigen spotting, the nitrocellulose membrane was incubated and antigens were blocked in three modes (Figure 2 and Figure 3). Our optimized conditions indicated that immobilization at damp thermosis for $2 \mathrm{~h}$ at $37^{\circ} \mathrm{C}$ and blocking with $5 \%$ skimmed milk powder ( $50 \mathrm{~g}$ skimmed milk powder dissolved in $1 \mathrm{~L}$ distilled water) for $1 \mathrm{~h}$ achieved suitable results. Antigen-loading concentrations and serum dilution were optimized using checkerboard titration. Results showed that the optimal loading concentrations of Eno, Fba1, and TR were $0.5 \mathrm{mg} / \mathrm{mL}, 0.04 \mathrm{mg} / \mathrm{mL}$, and $1 \mathrm{mg} / \mathrm{mL}$, respectively, with optimal serum dilutions at 1:10.

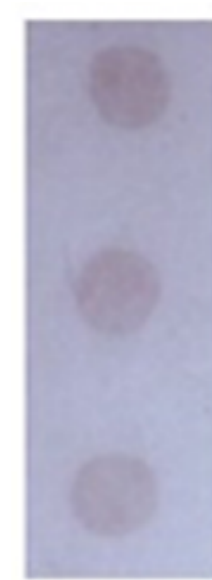

A

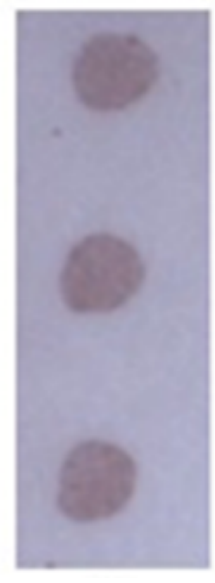

B

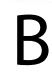

Figure 1: Comparison of different loading buffers for fixing Eno antigen. (A) PBS (pH 7.4). (B) Tris- $\mathrm{HCl}$ (pH 8.5). (C) CBS (pH 9.6).

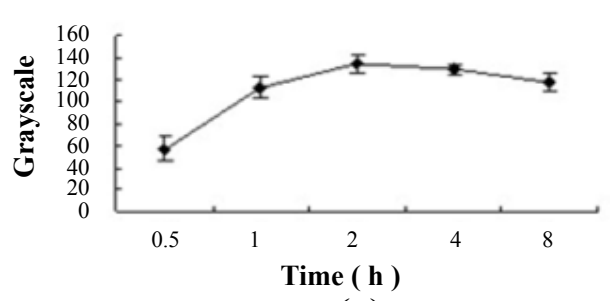

(a)

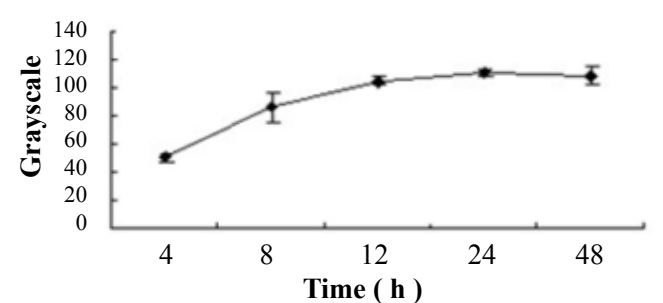

(b)

Figure 2: The effect of two antigen-coated conditions on Enoantigen detection. After spotting, the antigen was immobilized in two modes: 1 ) incubation at damp thermosis at $37^{\circ} \mathrm{C}$, where the gray value reached its peak following incubation for $2 \mathrm{~h}$, with a slight decrease at $8 \mathrm{~h}$; and 2 ) incubation at damp thermosis at $4^{\circ} \mathrm{C}$, where increase of the gray value was not observed following incubation for $>12 \mathrm{~h}$.

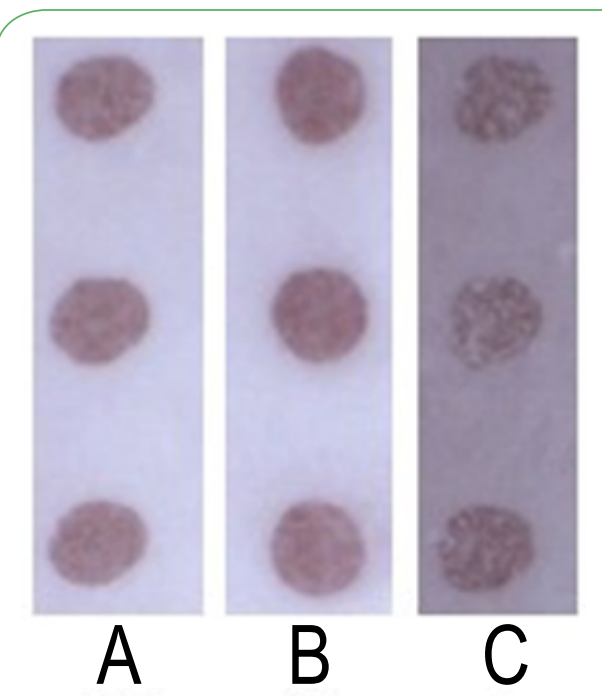

Figure 3: Comparison of different coating methods for Eno-antigen detection. (A) Incubation at $37^{\circ} \mathrm{C}$ for $2 \mathrm{~h}$. (B) Incubation at $4^{\circ} \mathrm{C}$ for $12 \mathrm{~h}$. (C) Drying at $37^{\circ} \mathrm{C}$ for $2 \mathrm{~h}$. After drying at $37^{\circ} \mathrm{C}$ for $2 \mathrm{~h}$, the blank area exhibited a high background, which was eliminated when incubated at damp thermosis for $2 \mathrm{~h}$ at $37^{\circ} \mathrm{C}$ or for $12 \mathrm{~h}$ at $4^{\circ} \mathrm{C}$.

\section{Specificity, stability of microarray}

Specificity: Blocking tests were conducted using serum detected as strongly positive for the antigens of interest according to ELISA. Sera were diluted to $1: 10$ and incubated with recombinant antigens at different concentrations $(0,0.5,1,2$, or $4 \mathrm{mg} / \mathrm{mL})$ for $1 \mathrm{~h}$ at $37^{\circ} \mathrm{C}$, and after drying, the microarray was scanned. The results suggested that when the final concentrations of recombinant Eno, Fba1, and TR reached $1 \mathrm{mg} / \mathrm{mL}, 0.5 \mathrm{mg} / \mathrm{mL}$, and $2 \mathrm{mg} / \mathrm{mL}$, respectively, the gray values from the scanned results were successively reduced from 188 to 33,130 to 13 , and 166 to 12 , respectively indicating that the testing results exhibited high specificity. All these results indicated the microarray devices leading to good repeatability.

Stability: Protein-microarray devices using the same samples preserved at $4^{\circ} \mathrm{C}$ under vacuum conditions were used to test antigen-positive sera and mixed sera from controls every 15 days, and the results were compared to those from freshly prepared devices scanned within $24 \mathrm{~h}$. The results indicated that the sensitivity and specificity of the test decreased gradually along with increased storage duration.

\section{Diagnostic value of the antibody test on IC and IA patients}

In the presence of patient sera, the antigen spot on the device was red, indicating the presence of the corresponding antibody (Figure 4). Based on the performance characteristics of the antiEno, -Fba1, and -TR antibodies, antigen detection in sera were assessed by ROC-curve analysis. AUC cutoff values of 66.5 and 29.5 for anti-Eno and -Fba1 antibodies in the serum samples were used to diagnose IC, returning 0.910 (95\% confidence interval (Cl), 0.899-0.920) and $0.880(\mathrm{Cl}, \sim 0.870-0.890)$, respectively (Figure 5A and Figure 5B). At cutoff values of 66.5 and 29.5, the specificity and sensitivity of the anti-Eno and -Fba1 antibodies were $88 \%$ and $87 \%$ and $77 \%$ and $86 \%$, respectively. At a cutoff 
of 58.5 for the anti-TR antibody in the serum samples used to diagnose IA, the AUC was $0.925(95 \% \mathrm{Cl}, \sim 0.916-0.934)$ (Figure $5 \mathrm{C})$, with a sensitivity of $78 \%$ and a specificity of $96 \%$. These results indicated that this method displayed superior diagnostic ability.

\section{Testing results using the anti-Eno and anti-Fba1 antibodies in serum from IC patients}

Serum from 105 patients with IC, 127 non-IC patients (including 42 IA patients, 50 cases of Candida colonization, 30 cases of bacteremia, and 5 patients with other fungal infections), and 183 healthy people was tested using the microarray devices. The anti-Eno antibody was detected in 71 cases (67.6\%), with median gray values of $85(49.5,142)$ as compared with those from healthy control and non-IC patients $(19(13,30.25), P<0.001$ and $21.5(16,29), P<0.001$, respectively). The median gray value for 68 cases (64.8\%) using the anti-Fba1 antibody was 36 (23.5, $72.5)$ as compared with those from healthy controls and nonIC patients $(13.5(10.0,18.0), P<0.001)$ and $21.0(15.0,27.0)$ $P<0.001$, respectively) (Figure 6 ). The positive rates of antibody detection of all forms of Candida infection are presented in Table 1 . The positive rates for antibody detection in sera from IC patients were significantly different from that observed for Candida colonization $(P<0.001)$. The positive rates for the anti-
Eno antibody in sera from patients infected with $C$. albicans and non- $C$. albicans were $76.9 \%(20 / 26)$ and $64.6 \%(51 / 79)$, respectively ( $\left.\chi^{2}=1.37, P>0.05\right)$, and for the anti-Fba1 antibody were $65.4 \%(17 / 26)$ and $64.6 \%(51 / 79)$, respectively $\left(\chi^{2}=0.006\right.$, $P>0.05)$. Additionally, compared with detection by anti-Eno or anti-Fba1 only, sensitivity and specificity improved to $81.0 \%$ and $89.0 \%$, respectively, with positive- and negative-predictive values of $71.4 \%$ and $93.2 \%$, respectively. When detection was undertaken using both antibodies, the results revealed higher sensitivity and negative-predictive values (Table 2).

\section{Testing results using the anti-TR antibody in serum from IA patients}

Serum from 42 IA patients, 190 non-IA patients (including 105 IC patients, 50 cases of Candida colonization, 30 cases of bacteremia, and 5 patients with other fungal infections), and 183 healthy individuals was tested using the microarray devices. The results in sensitivity and specificity of antibody detection were $71.4 \%$ (30/42) and 98.1\% (366/373), respectively. Thirty cases (71.4\%) were detected using the anti-TR antibody, with a median gray value of $130.0(25.8,218.8)$, whereas the median gray values for sera from healthy controls and non-IA patients at 28.0 (23.0, 32.0; $P<0.001)$ and $33.0(26.0,42.0 ; P<0.001)$, respectively (Figure 7). A total of 42 patients were detected by Aspergillus
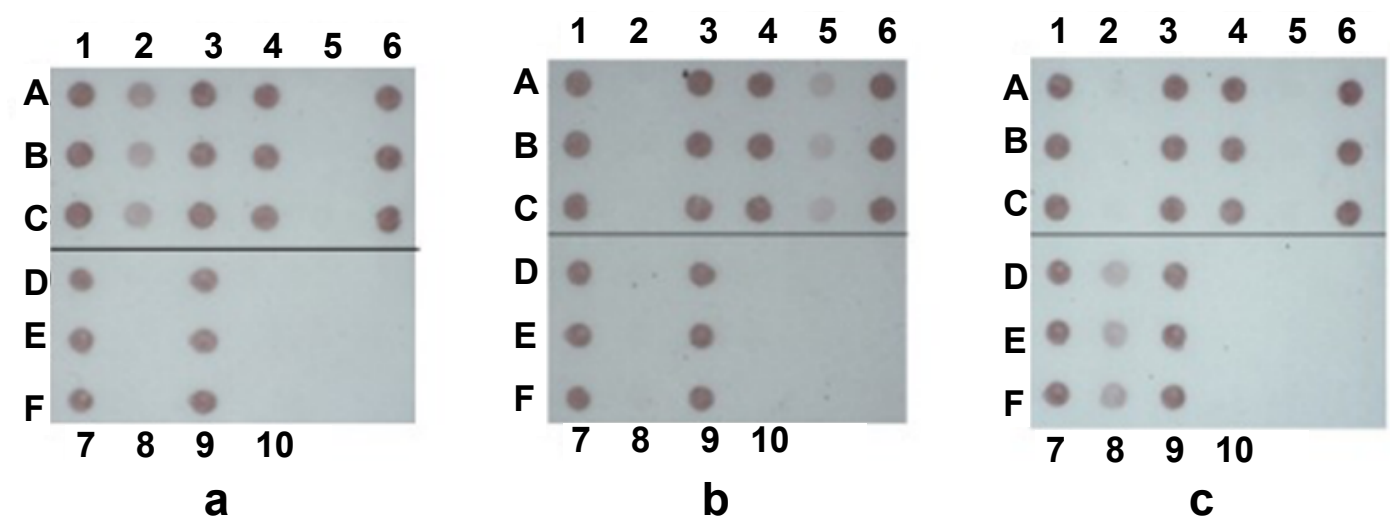

Figure 4: Protein microarray results. 1, 3, 4, 6, 7, and 9: human IgG; 2: purified Eno; 5: purified Fba1; 8: purified TR; 10: total proteins from Escherichia coli. (A) Positive results associated with the (A) anti-Eno, (B) anti-Fba1, and (C) anti-TR antibodies.

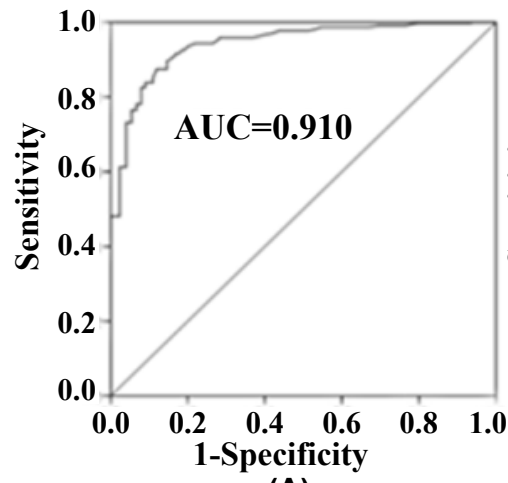

(A)

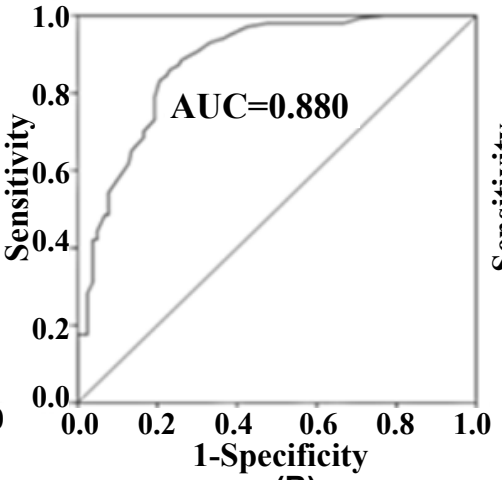

(B)

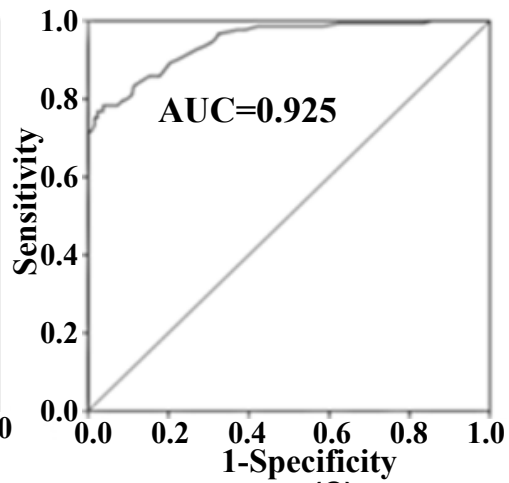

(C)

Figure 5: Receiver operating characteristic (ROC) curves for the anti-Eno, anti-Fba1, and anti-TR antibodies. ROC curves for the (A) anti-Eno, (B) anti-Fba1, and (C) anti-TR antibodies. 
A

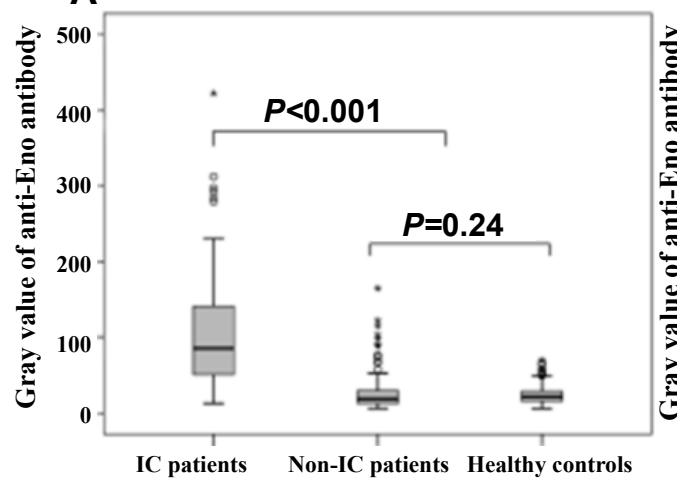

B

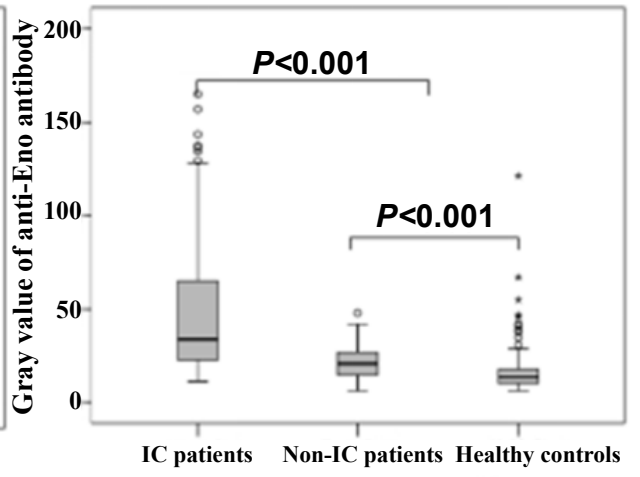

Figure 6: Protein microarray detection of anti-Eno and anti-Fba1 antibodies in the sera from IC patients and controls. (A) Anti-Eno antibody detection of Eno and (B) anti-Fba1 antibody detection of Fba1 in serum from IC patients and control groups. The gray values between IC patients and control groups were both considered statistically significant $(P<0.001)$ in terms of the anti-Eno and anti-Fba1 antibodies. Differences in the detection of the Eno antigen in non-IC patients and healthy controls were not significant $(P=0.240)$, whereas differences in Fba1 detection were significant $(P<0.001)$.

Table 1. Serological and microbiological surveillance of patients with IC and Candida colonization (n (\%)).

\begin{tabular}{|c|c|c|c|c|c|c|c|c|}
\hline \multirow{2}{*}{ Species } & \multicolumn{4}{|c|}{ Anti-Eno antibody } & \multicolumn{4}{c|}{ Anti-Fba1 antibody } \\
\cline { 2 - 11 } & \multicolumn{2}{|c|}{ IC patients } & Candida colonization & \multicolumn{3}{|c|}{ IC patients } & \multicolumn{2}{c|}{ Candida colonization } \\
\cline { 2 - 10 } & $n$ & Positive cases (\%) & $n$ & Positive cases (\%) & $n$ & Positive cases (\%) & $n$ & Positive cases (\%) \\
\hline Candida albicans & 26 & $20(76.9)$ & 37 & $6(16.2)$ & 26 & $17(65.4)$ & 37 & $3(8.1)$ \\
\hline Candida tropicalis & 19 & $9(47.4)$ & 5 & $1(20.0)$ & 19 & $10(52.6)$ & 5 & $3(60.0)$ \\
\hline Candida parapsilosis & 22 & $18(81.8)$ & - & - & 22 & $13(59.1)$ & - & - \\
\hline Candida glabrata & 10 & $7(70.0)$ & 3 & $2(66.7)$ & 10 & $10(100.0)$ & 3 & $1(33.3)$ \\
\hline Candida guilliermondii & 5 & $2(40.0)$ & - & - & 5 & $3(60.0)$ & - & - \\
\hline Other & 23 & $15(65.2)$ & 5 & $0(0)$ & 23 & $15(65.2)$ & 5 & $0(0)$ \\
\hline Total & 105 & $71(67.6)^{\text {a }}$ & 50 & $9(18.0)$ & 105 & $68(64.8)^{b}$ & 50 & $7(14.0)$ \\
\hline
\end{tabular}

Positive rates were compared against those of the respective Candida-colonization group $\left(\chi^{2}{ }_{\mathrm{a}}=33.39, P<0.001 ; \chi^{2}{ }_{\mathrm{b}}=34.95, P<0.001\right)$.

Table 2. Diagnostic value of anti-Eno and anti-Fba1 on the test cohort.

\begin{tabular}{|c|c|c|c|}
\hline & Detect anti-Eno antibody only & Detect anti-Fba1 antibody only & Combined detection \\
\hline True negative & 299 & 280 & 276 \\
\hline False positive & 11 & 30 & 34 \\
\hline True positive & 71 & 68 & 85 \\
\hline False negative & 34 & 37 & 20 \\
\hline Sensitivity (\%) & 67.6 & 64.8 & 81 \\
\hline Specificity (\%) & 96.5 & 90.3 & 89 \\
\hline NPV(\%) & 89.8 & 88.3 & 93.2 \\
\hline PPV (\%) & 86.5 & 69.3 & 71.4 \\
\hline
\end{tabular}

NPV: Negative-Predictive Value; PPV: Positive-Predictive Value

galactomannan simultaneously, with 22 positive cases at a sensitivity of only $52.4 \%$, which was significantly lower than that returned using the microarray chip to detect the anti-TR antibody $(P<0.010)$.

\section{Discussion}

The clinical diagnosis of IFI, a common infection that can cause death in immune-compromised patients, remains difficult. Recently, detection of fungal infections by serological methods through the recognition of fungal antigens, antibodies, and metabolites, has become an attractive field of study [17]. ELISA is currently used as a common serological-detection method; however, this method is time-consuming, labor-intensive, and requires large quantities of sample. Compared with the traditional methods, protein microarrays based on low-density microarray devices are advantageous because $[18,19]: 1$ ) they require small sample volumes and exhibit detection at the nanogram level; 2) they enable high-throughput, parallel analysis of thousands of protein samples within a single experiment; and 3) they exhibit a high signal-to-noise ratio.

Generally, expensive scanning equipment is essential for proteinmicroarray assays; however, the immune colloidal-gold technique 


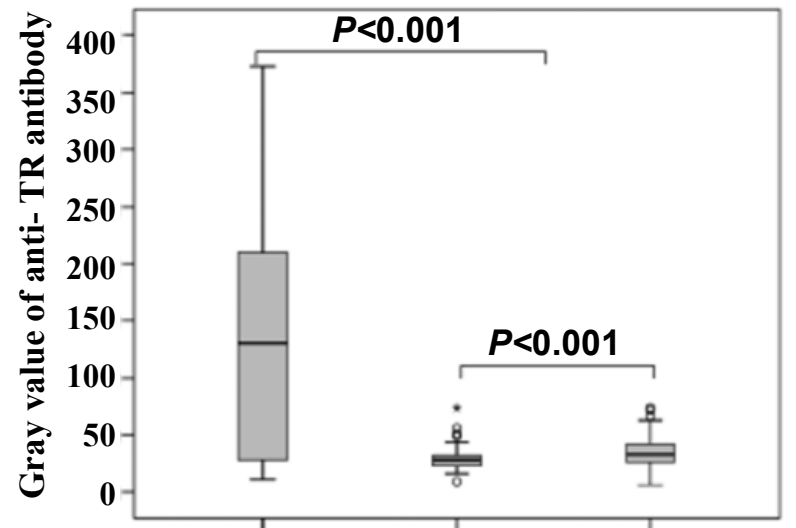

IA pateints Non-IA pateints Healthy controls

Figure 7: Protein microarray detection of the anti-TR antibody in sera from IA patients and controls. Differences in gray values derived from anti-TR antibody detection of the TR antigen between IApatient serum and that from controls were considered statistically significant $(P<0.001)$, as were differences between detection in serum from non-IA patients and healthy controls $(P<0.001)$.

avoids this limitation [20]. In this study, protein-microarray devices were constructed and optimized in combination with colloidal gold, thereby precluding the need for expensive scanning equipment.

Based on our optimized conditions, we prepared the proteinmicroarray devices to detect antigens in sera from IC and IA patients using three specific antibodies. Our results indicated that the methodology exhibited excellent specificity, repeatability, and stability and would meet the requirements of a clinical laboratory. These findings provide a foundation for further research in clinical settings to evaluate the technique for clinical applications.

Blood culture was used as the gold standard to assess the detection of sera from clinical patients using microarray devices. The positive detection rates for anti-Eno and -Fba1 antibodies were $67.6 \%(71 / 105)$ and $64.8 \%(68 / 105)$, respectively, for all Candida spp. in IC-patient sera and $71.4 \%$ (30/42) for anti-TR antibody in sera from 42 IA patients. Compared with ELISA, using microarray devices to detect anti-Eno, anti-Fba1, and anti-TR antibodies individually resulted in decreased sensitivity; however, combined detection of both anti-Eno and anti- Fba1 antibodies simultaneously increased the sensitivity to $81 \%$. Additionally, compared to previous ELISA results, the total coincidence rates associated with the protein-microarray method for the detection of anti-Eno and anti-Fbal antibodies were $89.5 \%$ and $77.1 \%$, respectively, with positive-coincidence rates of $89.5 \%$ and $83.6 \%$, respectively, and negative-coincidence rates of $89.7 \%$ and $96.0 \%$, respectively. Additionally, the consistency coefficient Kappa values were 0.751 and 0.685 , respectively. The total compliance rate of these two methods for detection of the anti-TR antibody was $88.1 \%$, with a positive-coincidence rate of $88.2 \%$, a negativecoincidence rate of $87.5 \%$, and a consistency coefficient Kappa of 0.662 . These results indicated that the two methods were consistent in the determination of anti-Eno, anti-Fba1, and anti-TR antibodies, suggesting that the detection of dominant antibodies from Candida and Aspergillus infections by protein microarray can also provide rapid and effective etiological diagnosis of IFI.

Our results indicated that joint detection improved diagnostic sensitivity, but reduced specificity, which is required to exclude false-positive diagnosis. Suspicious positive results derived from the application of low-density protein microarrays should be retested using ELISA to confirm test results. However, all methods should be combined with investigation of patient medical history and laboratory, radiological, and histopathologic examinations to ensure a correct diagnosis.

\section{Conclusion}

This study described a new diagnostic method for detecting associated antibodies from Candida and Aspergillus infections, providing a foundation for future work in this area. Additionally, some previously reported immunodominant antigens capable of use in the diagnosis of IFI, such as histone methyltransferase

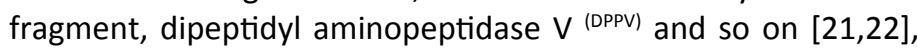
were not included in this microarray device; therefore, this method requires further improvement by increasing the use of other dominant antigens to enhance diagnostic sensitivity and accuracy.

\section{Acknowledgments}

This work was supported financially by the Jiangsu Science Foundation (BE2009673).

\section{Author contributions}

$\mathrm{XC}$ and CFM contributed to the analysis and interpretation of data and drafted the manuscript. FQL conceived, coordinated, and designed the study, LNS and YAH performed the experiments and was involved in drafting the article. All the authors have read and approved the final manuscript. 


\section{References:}

1 Morrissey CO (2013) Advancing the Field: Evidence for New Management Strategies in Invasive Fungal Infections. Curr Fungal Infect Rep 7: 51-58.

2 De Bernardis F, Arancia S, Sandini S, Graziani S, Norelli S (2015) Studies of Immune Responses in Candida vaginitis. Pathogens 4: 697-707.

3 Singh S, Fatima Z, Hameed S (2015) Predisposing factors endorsing Candida infections. Infez Med 23: 211-213.

4 Arvanitis M, Mylonakis E (2015) Diagnosis of Invasive Aspergillosis: Recent Developments and Ongoing Challenges. Eur J Clin Invest 45 646-652.

5 Sipsas NV, Kontoyiannis DP (2012) Invasive fungal infections in patients with cancer in the intensive care unit. Int J Antimicrob Agents 39: 464-471.

6 Farmakiotis D, Kontoyiannis DP (2015) Emerging Issues With Diagnosis and Management of Fungal Infections in Solid Organ Transplant Recipients. Am J Transplant 15: 1141-1147.

7 Mutschlechner W, Risslegger B, Willinger B (2015) Bronchoalveolar Lavage Fluid (1,3)B-D-Glucan for the Diagnosis of Invasive Fungal Infections in Solid Organ Transplantation:A Prospective Multicenter Study. Transplantation 99: e140-e144.

8 Miceli MH, Maertens J (2015) Role of Non-Culture-Based Tests, with an Emphasis on Galactomannan Testing for the Diagnosis of Invasive Aspergillosis. Semin Respir Crit Care Med 36: 650-661.

9 Templin MF, Stoll D, Schwenk JM, Pötz O, Kramer S, et al. (2003) Protein microarrays: promising tools for proteomic research. Proteomics 3: 2155-2166.

10 Poetz O, Schwenk JM, Kramer S, Stoll D, Templin MF, et al. (2005) Protein microarrays: catching the proteome. Mech Ageing Dev 126: 161-170.

11 Richens JL, Lunt EA, O'Shea P (2015) Optimisation of proteinmicroarray techniques for analysis of the plasma proteome: Minimisation of nonspecific binding interactions. Int Immunopharmacol 24: 166-168.

12 Laín A, Elguezabal N, Amutio E (2008) Use of recombinant antigens for the diagnosis of invasive candidiasis. Clin Dev Immunol 2008: 721-950.

13 Shi LN, Li FQ, Lu JF (2012) Antibody specific to thioredoxin reductase as a new biomarker for serodiagnosis of invasive aspergillosis in nonneutropenic patients. Clin Chim Acta 413: 938-943.

14 Li FQ, Ma CF, Shi LN (2013) Diagnostic value of immunoglobulin G antibodies against Candida enolase and fructose-bisphosphate aldolase for candidemia. BMC Infect Dis 13: 253.

15 Shi LN, Li FQ, Huang M (2012) Immunoproteomics based identification of thioredoxin reductase GliT and novel Aspergillus fumigatus antigens for serologic diagnosis of invasive aspergillosis. BMC Microbiology 12: 11.

16 De Pauw B, Walsh TJ, Donnelly JP (2008) Revised definitions of invasive fungal disease from the European Organization for Research and Treatment of Cancer/Invasive Fungal Infections Cooperative Group and the National Institute of Allergy and Infectious Diseases Mycoses Study Group (EORTC/MSG) Consensus Group. Clin Infect Dis 46: $1813-1821$.

17 Sendid B, Caillot D, Baccouch-Humbert B (2003) Contribution of the Platelia Candida-specific antibody and antigen tests to early diagnosis of systemic Candida tropicalis infection in neutropenic adults. J Clin Microbiol 41: 4551-4558.

18 Zhu H, Klemic JF, Chang S (2000) Analysis of yeast protein kinases using protein chips. Nat Genet 26: 283-289.

19 Cahill DJ (2001) Protein and antibody arrays and their medical applications. J Immunol Methods 250: 81-91.

20 Zhao YH, Wang XR, Chen PC (2015) Detection of Antibodies against Avian Influenza Virus by Protein Microarray using Nucleoprotein Expressed in Insect Cells. J Vet Med Sci 77: 413-419.

21 Clancy CJ, Nguyen M-L, Cheng S (2008) Immunoglobulin G responses to a panel of Candida albicans antigens as accurate and early markers for the presence of systemic candidiasis. J Clin Microbiol 46: 16471654.

22 Kumar A, Ahmed R, Singh PK, Shukla PK (2011) Identification of virulence factors and diagnostic markers using immunosecretome of Aspergillus fumigatus. J Proteomics 74: 1104-1112. 